\title{
ConA-based glucose sensing using the long-lifetime Azadioxatriangulenium fluorophore
}

\author{
Brian Cummins ${ }^{\mathrm{a}, \mathrm{b}, \mathrm{e}^{*},}$, Jonathan Simpson ${ }^{\mathrm{b}}$, Zygmunt Gryczynski ${ }^{\mathrm{c}}$, Thomas Just Sørensen ${ }^{\mathrm{d}}$, Bo W \\ Laursen $^{\mathrm{d}}$, Duncan Graham ${ }^{\mathrm{b}}$, David Birch ${ }^{\mathrm{e}}$, Gerard Coté ${ }^{\mathrm{a}}$ \\ ${ }^{a}$ Department of Biomedical Engineering, Texas A\&M University, College Station, TX, USA, 77843 \\ ${ }^{\mathrm{b}}$ Department of Pure and Applied Chemistry, WestCHEM, University of Strathclyde, Glasgow, UK, \\ G1 1XL \\ ${ }^{\mathrm{c}}$ Department of Physics and Astronomy, Texas Christian University, Fort Worth, TX, USA and \\ Center for Commercialization of Fluorescence Technologies, Department of Molecular Biology and \\ Immunology, University of North Texas Health Science Center, Fort Worth, TX, USA \\ ${ }^{\mathrm{d}}$ Nano-Science Center \& Department of Chemistry, University of Copenhagen, Universitetsparken \\ 5, DK-2100 København Ø, Denmark \\ ${ }^{\mathrm{e}}$ Department of Physics, University of Strathclyde, Glasgow, UK, G1 1XQ
}

\begin{abstract}
:
Fluorescent glucose sensing technologies have been identified as possible alternatives to current continuous glucose monitoring approaches. We have recently introduced a new, smart fluorescent ligand to overcome the traditional problems of ConA-based glucose sensors. For this assay to be translated into a continuous glucose monitoring device where both components are free in solution, the molecular weight of the smart fluorescent ligand must be increased. We have identified ovalbumin as a naturally-occurring glycoprotein that could serve as the core-component of a $2^{\text {nd }}$ generation smart fluorescent ligand. It has a single asparagine residue that is capable of displaying an N-linked glycan and a similar isoelectric point to ConA. Thus, binding between ConA and ovalbumin can potentially be monovalent and sugar specific. This work is the preliminary implementation of fluorescently-labeled ovalbumin in the ConA-based assay. We conjugate the red-emitting, long-lifetime azadioxatriangulenium $\left(\mathrm{ADOTA}^{+}\right)$dye to ovalbumin, as ADOTA have many advantageous properties to track the equilibrium binding of the assay. The ADOTA-labeled ovalbumin is paired with Alexa Fluor 647-labeled ConA to create a Förster Resonance Energy Transfer (FRET) assay that is glucose dependent. The assay responds across the physiologically relevant glucose range $(0-500 \mathrm{mg} / \mathrm{dL})$ with increasing intensity from the ADOTA-ovalbumin, showing that the strategy may allow for the translation of the smart fluorescent ligand concept into a continuous glucose monitoring device.
\end{abstract}

Keywords: Glucose sensing, fluorescence, energy transfer, competitive binding

\section{INTRODUCTION}

Diabetes is a disease that is characterized by blood glucose concentrations that are consistently above normal, due to either the inability of the patient's pancreatic islet cells to make insulin (Type 1) or the resistance of their cells to respond to insulin (Type 2). More than 300 million people worldwide currently have diabetes, and those numbers are expected to rise. ${ }^{1}$ The elevated glucose concentrations increase the degree of non-enzymatic protein glycation and, as a result, cause secondary complications in the liver, heart, and kidney. 
In 1993, the Diabetes Control and Complications Trial reported 10 years of clinical data that showed that strict management of glucose concentrations within normal levels minimizes these complications. ${ }^{2}$ This included the frequent measurement of blood sugar concentrations and the appropriate intervention (diet, exercise, medication, and insulin). Approximately $10 \%$ of the diabetic population has Type 1 diabetes, which can be successfully controlled with the administration of insulin. The rest of the diabetic population has Type 2 diabetes, which primarily relies on the other interventions to control their blood sugar. Diabetes is often diagnosed by a glycated hemoglobin percentage (HbA1c) value of greater than $7 \% .^{3}$ Uncontrolled diabetes typically shows an AbA1c value of at least $9 \%$, corresponding to an estimated average glucose of $\sim 212 \mathrm{mg} / \mathrm{dL} .^{2}$ On average, if the patient can decrease their eAG by $30 \mathrm{mg} / \mathrm{dL}$, they decrease the risk of developing secondary complications by 40\%. The American Diabetes Association (ADA) suggests that the target set for most diabetic patients should be an HbA1c value of $7 \%$ (corresponding to an eAG of $154 \mathrm{mg} / \mathrm{dL}$ ). ${ }^{3}$

The primary method of measuring the blood sugar is via the blood glucose meter, and it is recommended that patients make 7 glucose measurements per day. However, $60 \%$ of Type 1 diabetic patients average less than a single reading per day. ${ }^{4}$ Continuous glucose monitors (CGM) offer the patient more information and open the possibility for improved glycemic control. While current commercially available CGM primarily can be used in an open-loop system, CGM devices are expected to be instrumental in the development of the closed-loop glucose control known as the 'artificial pancreas.' This closed-loop control is expected to have a major impact on the treatment of Type 1 diabetes. ${ }^{5}$

The current, commercially-available continuous glucose monitoring devices all use the electrochemical enzymatic approach. These $1^{\text {st }}$ generation enzymatic sensors rely on oxygen diffusion. This enzymatic based system consumes the glucose, and the byproducts are detected by the electrode. ${ }^{6}$ This type of chemistry has shown to be accurate and repeatable during in vitro tests. However, it requires frequent calibrations for accurate predictions when inserted underneath the skin in the percutaneous approach. Because the sensor measures the byproducts from the oxygenmediated glucose consumption, it is highly sensitive to the foreign body response of the host. This host response changes the glucose and oxygen transport from the interstitial fluid to the sensor and shifts the reported values by the sensor. Even with calibrations, current sensors must be replaced after 3-7 days. ${ }^{7}$

A large research effort is put towards finding alternative continuous glucose monitoring sensors that may avoid some of these drawbacks to the traditional approach. ${ }^{8}$ One approach is an affinity-based assay that transduces the signal via fluorescence. ${ }^{9}$ Our group has pursued an affinity-based sensing system that has paired the glucose and mannose binding lectin, Concanavalin A (ConA), with a high-affinity fluorescent ligand to which it binds. ${ }^{10-15}$ The binding of ConA to the fluorescent ligand can be engineered to respond to increasing concentrations of glucose, and this can be tracked via fluorescence with either anisotropy or energy transfer. This assay has traditionally shown problems with irreversibility and precipitation. ${ }^{16-18}$ However, we have recently overcome these obstacles in free solution by introducing a smart fluorescent ligand. This smart fluorescent ligand: (1) displays a single moiety that can bind to ConA's full binding site, (2) is negatively charged, and (3) is fluorescently labeled. ${ }^{19}$ An obstacle remains for this strategy to be used in a continuous glucose sensor: the size of this smart fluorescent ligand must be increased to prevent leaching from the semipermeable membrane that allows for glucose diffusion.

In this work we present a preliminary study that introduces ovalbumin (the primary protein in egg-white) as the highaffinity fluorescent ligand in a ConA-based glucose sensing assay. Ovalbumin has been identified as a possible $2^{\text {nd }}$ generation smart fluorescent ligand as: (1) it has a single glycosylation site that can present a high-mannose glycan, (2) it is negatively charged at physiological $\mathrm{pH}$ (with an isoelectric point of 4.5), (3) it has a molecular weight of $45 \mathrm{kD}$, and (4) it has numerous lysine residues that can be labeled with an amine-reactive fluorophore. This preliminary study uses the Azadioxatriangulenium $\left(\mathrm{ADOTA}^{+}\right.$) fluorophore as $\mathrm{ADOTA}^{+}$has fluorescence properties ideal for tracking the binding between ovalbumin and ConA. ${ }^{20-22}$ ADOTA $^{+}$displays excitation and emission maxima at $540 \mathrm{~nm} / 560 \mathrm{~nm}$ with a fluorescence lifetime of $\sim 23 \mathrm{~ns}$. It has moderate brightness with a molar extinction coefficient of $9,800 \mathrm{~cm}^{-1} \mathrm{M}^{-1}$ and a quantum yield of 0.49 (determined in acetonitrile), with an intrinsic anisotropy has been shown to be $0.38 .^{23,24}$ 
Therefore, $\mathrm{ADOTA}^{+}$can allow binding studies between relatively large molecules to be tracked with anisotropy, ${ }^{25}$ and it can be used to avoid the shorter fluorescence lifetimes that are common for endogenous fluorophores. ${ }^{26}$ Figure 1 shows a schematic of the energy transfer from the ADOTA-labeled glycated ovalbumin to the Alexa Fluor 647-labeled ConA.

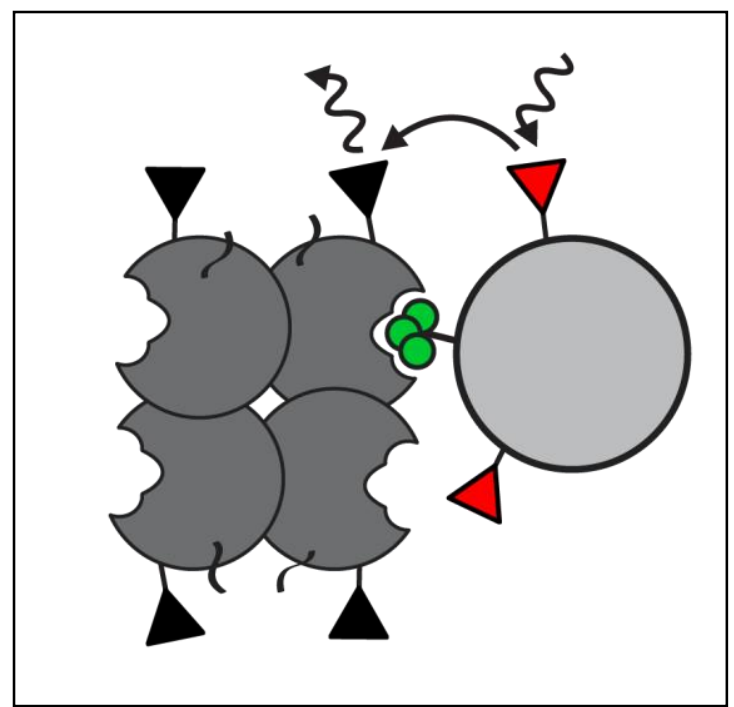

Figure 1: Schematic of the energy transfer from the ADOTA fluorophore (red triangle) to the AF647 fluorophore (black triangle) upon binding of the ovalbumin (light gray) to the ConA (dark gray tetramer) via the single N-linked glycan (green).

\section{MATERIALS \& METHODS}

Alexa Fluor 647-labeled Concanavalin A (AF647-ConA) was purchased from Invitrogen (Grand Island, NY). Ovalbumin, mannose, TRIS buffer, $\mathrm{NaCl}, \mathrm{MnCl} 2, \mathrm{CaCl} 2$, sodium bicarbonate, DMSO, were purchased from Sigma (St. Louis, MO) and used as received. The ADOTA-NHS was received from Dr. Thomas Just Sørensen and Dr. Bo W Laursen (København, Dk). ${ }^{24-26}$ TRIS buffer was prepared to be $0.01 \mathrm{M}$ TRIS, $0.15 \mathrm{M} \mathrm{NaCl}, 0.001 \mathrm{M} \mathrm{MnCl}_{2}, 0.001 \mathrm{M}$ $\mathrm{CaCl}_{2}, \mathrm{pH} 7.4$.

The ConA-based assay, as we present it, tracks the fluorescence intensity from the fluorescently-labeled ovalbumin. As a result, it is essential that all of the ovalbumin can bind to ConA. If a portion of the fluorescently labeled ovalbumin cannot bind to ConA, it will serve as a background signal that is not sensitive to glucose concentration and will increase the error of the sensor. The glycans on proteins can display a significant amount of variation, and a large fraction of proteins that can be glycated do not actually display glycans.

We separated the glycated fraction from the non-glycated fraction of our ovalbumin sample by performing affinitychromatography with ConA-functionalized resin. The protein elution from the column was tracked by monitoring the absorbance at $280 \mathrm{~nm}$. After the non-glycated fraction eluted from the column, high concentrations of mannose were used to elute the glycated fraction. This glycated fraction was collected and dialyzed in sodium bicarbonate buffer to prepare the sample for labeling.

This glycated fraction of the ovalbumin was labeled with the ADOTA-NHS using traditional amine-reactive protocol. Briefly, ADOTA-NHS was dissolved in DMSO, added dropwise to the solution of glycated ovalbumin in sodium bicarbonate buffer ( $\mathrm{pH} 9$ to deprotonate the primary amines), mixed well, and allowed to react for 1 hour in the dark at 
room temperature. Afterwards, the free dye was removed via dialysis against TRIS buffer, and the solution was filtered with syringe filters. UV/VIS absorbance measurements were performed in TRIS buffer. Using the molar extinction coefficients, the concentration and degree of labeling of the ADOTA-ovalbumin was determined. Similar UV/VIS studies were performed with the AF647-ConA that was purchased from Invitrogen. Characteristic excitation and emission spectra are shown in Figure 2.

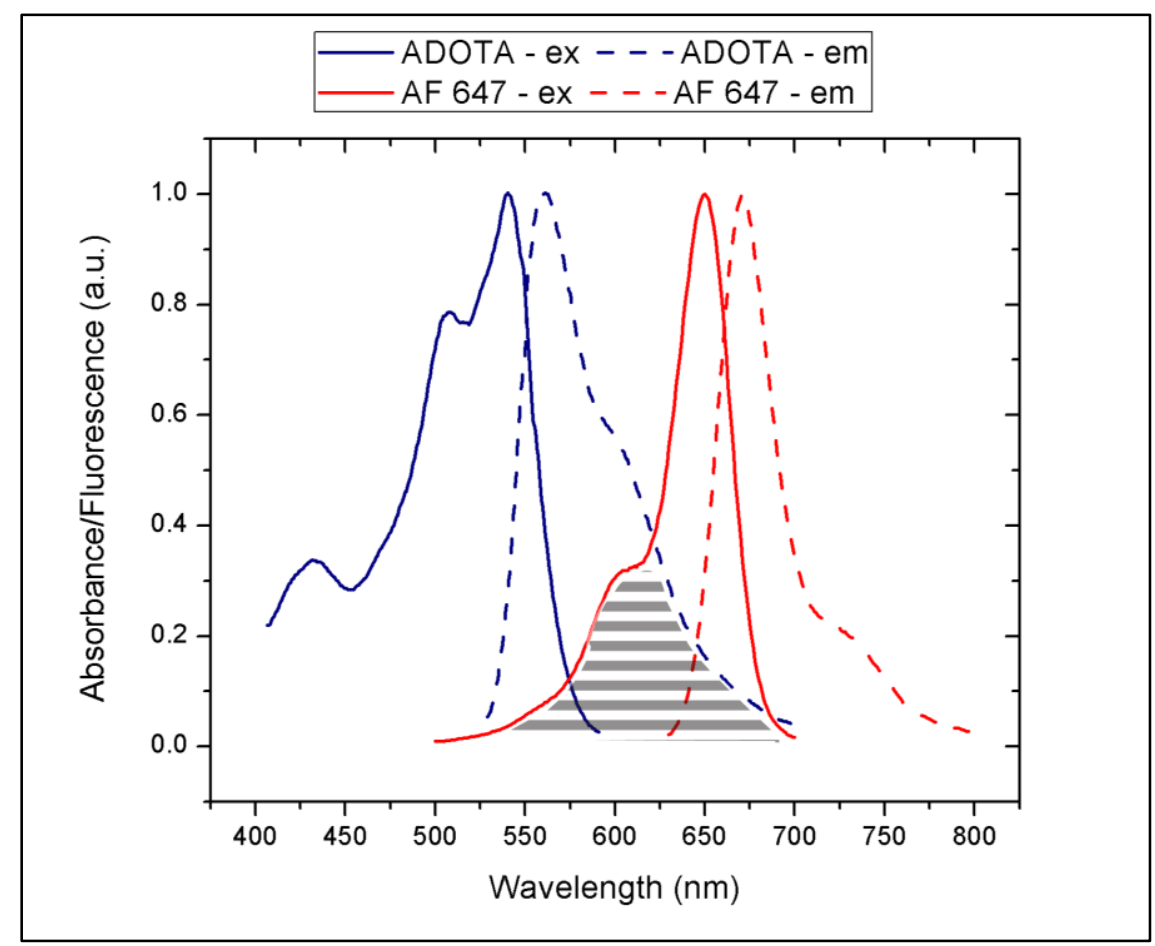

Figure 2: Excitation/Emission spectra of $\mathrm{ADOTA}^{+}$and AF 647 in TRIS. The dashed horizontal line -indicates the spectral overlap which is required for energy transfer.

The assay was chosen to have $500 \mathrm{nM}$ ADOTA-ovalbumin and $1 \mu \mathrm{M}$ AF647-ConA, and was loaded into a cuvette of TRIS buffer. Highly concentrated glucose aliquots were added to minimize dilution effects. Upon each addition, the solutions were mixed well and given time to equilibrate. At each glucose concentration $(0 \mathrm{mg} / \mathrm{dL}-500 \mathrm{mg} / \mathrm{dL}))$, the steady-state fluorescence was measured on a Fluorolog 3. Excitation was at $500 \mathrm{~nm}$ with a $10 \mathrm{~nm}$ bandwidth. Emission was collected from $530 \mathrm{~nm}$ to $750 \mathrm{~nm}$ with a $5 \mathrm{~nm}$ bandwidth.

\section{RESULTS \& DISCUSSION}

The degree of labeling of the ADOTA-ovalbumin was determined to be approximately 1.5 ADOTA fluorophores per glycated ovalbumin. The degree of labeling of the AF647-ConA was determined to be approximately 3 AF 647 fluorophores per ConA.

The assay displayed glucose-dependent fluorescence spectra that are characteristic of competitive binding (Figure 3a). The fluorescence of the ADOTA-ovalbumin increased with increasing concentration, indicating that the concentration of 
ADOTA-ovalbumin undergoing FRET (bound population) was decreasing. The emission of each fluorophore is sufficiently separated to minimize the effect that the changing fluorescence intensity of ADOTA has on the AF 647 fluorescence. The ratio of the donor to acceptor maxima $(560 \mathrm{~nm}$ to $670 \mathrm{~nm})$ as a function of glucose concentration is shown in Figure 3b. This shows that the fluorescence of this ADOTA-ovalbumin and AF 647 ConA assay primarily changes from 0-300 $\mathrm{mg} / \mathrm{dL}$, and then begins to flatten out. The primary region of interest for physiological glucose sensing is $40 \mathrm{mg} / \mathrm{dL}$ to $400 \mathrm{mg} / \mathrm{dL}$

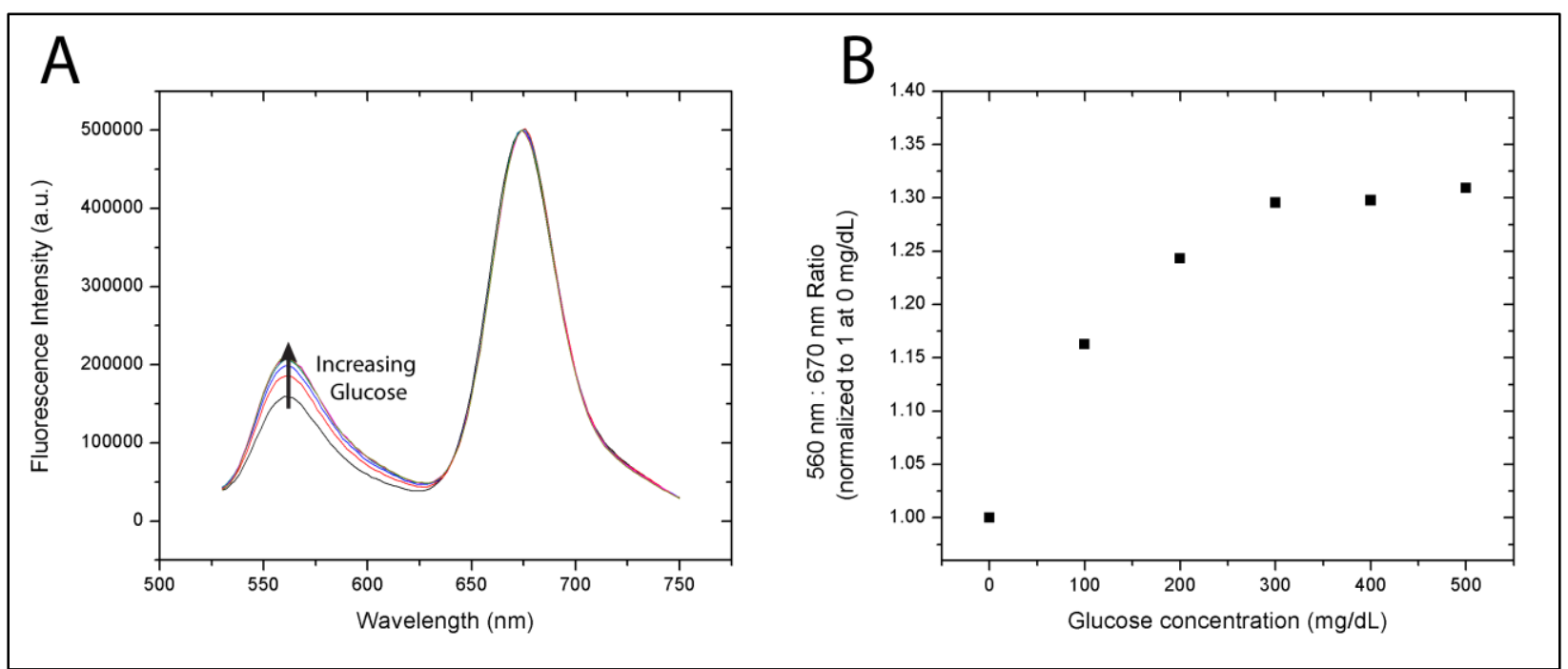

Figure 3: The fluorescence glucose response of an assay comprised of $500 \mathrm{nM}$ ADOTA-ovalbumin and $1 \mu \mathrm{M}$ AF647-ConA.

Improvements could potentially be made on the assay by increasing the degree of labeling on ConA and performing sitespecific labeling on the ovalbumin with the $\mathrm{ADOTA}^{+}$fluorophore. Both of these strategies could improve the average FRET efficiency of the bound ovalbumin population.

\section{CONCLUSION}

This work introduces the naturally-occurring protein, ovalbumin, as the core-component of a $2^{\text {nd }}$ generation smart fluorescent ligand to be paired with ConA in a fluorescence glucose sensor. The glycated fraction of ovalbumin was separated with affinity chromatography and labeled with the long-fluorescence-lifetime ADOTA $^{+}$dye to allow the binding to be tracked via fluorescence. A Förster Resonance Energy Transfer assay was generated by pairing ADOTAovalbumin with AF647-ConA that showed a fluorescence response across physiologically relevant glucose concentrations. This preliminary work indicates that the ADOTA-ovalbumin fluorescent ligand could potentially allow the translation of the smart fluorescent ligand concept into a continuous glucose monitoring device. 


\section{ACKNOWLEDGEMENTS}

The authors would like to thank the Whitaker Foundation for the Summer Grant to allow for this collaborative work.

\section{REFERENCES}

[1] G. Danaei, M. M. Finucane, Y. Lu et al., "National, regional, and global trends in fasting plasma glucose and diabetes prevalence since 1980: Systematic analysis of health examination surveys and epidemiological studies with 370 country-years and $2 \cdot 7$ million participants," The Lancet, 378(9785), 31-40 (2011).

[2] "The effect of intensive treatment of diabetes on the development and progression of long-term complications in insulin-dependent diabetes mellitus," New England Journal of Medicine, 329(14), 977-986 (1993).

[3] "Standards of medical care in diabetes - 2013," Diabetes Care, 36(SUPPL.1), S11-S66 (2013).

[4] A. M. Delamater, "Improving patient adherence," Clinical Diabetes, 24(2), 71-72+75-77 (2006).

[5] B. P. Kovatchev, E. Renard, C. Cobelli et al., "Feasibility of outpatient fully integrated closed-loop control first studies of wearable artificial pancreas," Diabetes Care, 36(7), 1851-1858 (2013).

[6] J. Wang, "Electrochemical glucose biosensors," Chemical Reviews, 108(2), 814-825 (2008).

[7] N. Wisniewski, F. Moussy, and W. M. Reichert, "Characterization of implantable biosensor membrane biofouling,” Fresenius' Journal of Analytical Chemistry, 366(6-7), 611-621 (2000).

[8] N. S. Oliver, C. Toumazou, A. E. G. Cass et al., "Glucose sensors: A review of current and emerging technology," Diabetic Medicine, 26(3), 197-210 (2009).

[9] J. C. Pickup, F. Hussain, N. D. Evans et al., "Fluorescence-based glucose sensors," Biosensors and Bioelectronics, 20(12), 2555-2565 (2005).

[10] J. S. Schultz, S. Mansouri, and I. J. Goldstein, "Affinity sensor: A new technique for developing implantable sensors for glucose and other metabolites," Diabetes Care, 5(3), 245-253 (1982).

[11] R. J. Russell, M. V. Pishko, C. C. Gefrides et al., "A fluorescence-based glucose biosensor using concanavalin A and dextran encapsulated in a poly(ethylene glycol) hydrogel," Analytical Chemistry, 71(15), 3126-3132 (1999).

[12] R. Ballerstadt, and J. S. Schultz, "A fluorescence affinity hollow fiber sensor for continuous transdermal glucose monitoring," Analytical Chemistry, 72(17), 4185-4192 (2000).

[13] B. L. Ibey, H. T. Beier, R. M. Rounds et al., "Competitive binding assay for glucose based on glycodendrimerfluorophore conjugates," Analytical Chemistry, 77(21), 7039-7046 (2005).

[14] B. M. Cummins, J. Lim, E. E. Simanek et al., "Encapsulation of a concanavalin A/dendrimer glucose sensing assay within microporated poly (ethylene glycol) microspheres," Biomedical Optics Express, 2(5), 1243-1257 (2011).

[15] B. M. Cummins, J. T. Garza, and G. L. Coté, "Optimization of a concanavalin A-based glucose sensor using fluorescence anisotropy,” Analytical Chemistry, 85(11), 5397-5404 (2013).

[16] L. Tolosa, H. Malak, G. Raob et al., "Optical assay for glucose based on the luminescnence decay time of the long wavelength dye Cy5 ${ }^{\mathrm{TM}}$," Sensors and Actuators, B: Chemical, 45(2), 93-99 (1997).

[17] L. J. McCartney, J. C. Pickup, O. J. Rolinski et al., "Near-infrared fluorescence lifetime assay for serum glucose based on allophycocyanin-labeled concanavalin A," Analytical Biochemistry, 292(2), 216-221 (2001).

[18] B. M. Cummins, J. T. Garza, and G. L. Coté, "Limitations of current fluorescent glucose sensing assays based on competitive binding." 8591.

[19] B. M. Cummins, M. Li, A. K. Locke et al., "A smart fluorescent ligand to overcome the aggregation problems of Concanavalin A-based glucose sensors," Submitted, (2014).

[20] B. W. Laursen, and F. C. Krebs, "Synthesis of a triazatriangulenium salt," Angewandte Chemie - International Edition, 39(19), 3432-3434 (2000).

[21] F. C. Krebs, "Synthesis, structure, and properties of azatriangulenium salts," Chemistry - A European Journal, 7(8), 1773-1783 (2001).

[22] S. Dileesh, and K. R. Gopidas, "Photoinduced electron transfer in azatriangulenium salts," Journal of Photochemistry and Photobiology A: Chemistry, 162(1), 115-120 (2004).

[23] E. Thyrhaug, T. J. Sørensen, I. Gryczynski et al., "Polarization and symmetry of electronic transitions in long fluorescence lifetime triangulenium dyes," Journal of Physical Chemistry A, 117(10), 2160-2168 (2013). 
[24] B. P. Maliwal, R. Fudala, S. Raut et al., "Long-Lived Bright Red Emitting Azaoxa-Triangulenium Fluorophores," PLoS ONE, 8(5), (2013).

[25] T. J. Sørensen, E. Thyrhaug, M. Szabelski et al., "Azadioxatriangulenium: a long fluorescence lifetime fluorophore for large biomolecule binding assay," Methods and Applications in Fluorescence, 1(2), 025001 (2013).

[26] R. M. Rich, D. L. Stankowska, B. P. Maliwal et al., "Elimination of autofluorescence background from fluorescence tissue images by use of time-gated detection and the AzaDiOxaTriAngulenium (ADOTA) fluorophore," Analytical and Bioanalytical Chemistry, 405(6), 2065-2075 (2013). 